\title{
Prediction of Second Neurological Attack in Patients with Clinically Isolated Syndrome using Support Vector Machines
}

\author{
Viktor Wottschel, Olga Ciccarelli, \\ Declan T. Chard and David H. Miller \\ Institute of Neurology, UCL \\ London, United Kingdom \\ Email:viktor.wottschel.12@ucl.ac.uk
}

\author{
Daniel C. Alexander \\ Dept. of Computer Science, UCL \\ London, United Kingdom
}

\begin{abstract}
The aim of this study is to predict the conversion from cinically isolated syndrome to clinically definite multiple sclerosis using support vector machines. The two groups of converters and non-converters are classified using features that were calculated from baseline data of 73 patients. The data consists of standard magnetic resonance images, binary lesion masks, and clinical and demographic information. 15 features were calculated and all combinations of them were iteratively tested for their predictive capacity using polynomial kernels and radial basis functions with leave-one-out cross-validation. The accuracy of this prediction is up to $86.4 \%$ with a sensitivity and specificity in the same range indicating that this is a feasible approach for the prediction of a second clinical attack in patients with clinically isolated syndromes, and that the chosen features are appropriate. The two features gender and location of onset lesions have been used in all feature combinations leading to a high accuracy suggesting that they are highly predictive. However, it is necessary to add supporting features to maximise the accuracy.
\end{abstract}

Keywords-Multiple Sclerosis; Clinically Isolated Syndrome; Support Vector Machine; Classifcation

\section{INTRODUCTION}

Multiple Sclerosis is a neurodegenerative disease characterised by inflammation, demyelination and axon degeneration leading to a disturbed signal transmission in the affected axons [1]. A first neurological attack typically leads to the diagnosis of a clinically isolated syndrome (CIS). It is often associated with lesions which are visible as hyperintense spots in the white matter (WM) structure of the brain in standard magnetic resonance imaging (MRI) protocols like T2- and PD-weighting [2],[4]. Depending on the onset lesion location disorders can affect e.g. vision, movement, or sensation [3]. In approximately $30 \%$ of the CIS patients a second neurological attack will occur during one year leading to the diagnosis of clinically definite multiple sclerosis (CDMS). At the same time, however, 20\% will not convert to CDMS even after 20 years [4]. The ability to predict the risk of a second neurological attack on a short-time basis at the time of presentation is of special interest for both the patient and the physician's treatment plan.

In a previous study [5] lesional features were defined, and the predicitive capacity of support vector machines
(SVMs) was examined using individual features and feature combinations calculated from onset data. The aim was the prediction of conversion from CIS to CDMS within one year. It was shown that combinations perform better and lead to accuracies of $77 \%$. Further work added demographic features and increased the classification accuracy to $84 \%$ [6]. Here, we present the results for a completely re-defined feature set based on the findings of the previous projects. The new feature definitions obviate the time-consuming registration of the images and, at the same time, remove a possible bias.

\section{Methods}

15 features were calculated from data of 73 patients who were scanned on the same MRI scanner in London. The scanning protocols included T1-, T2-, and PD-weighting, and lesions were manually masked from the PD-weighted scans by an experienced neurologist using semiautomated methods (Figure 1). Finally, clinically and demographic features were added.

The feature definitions are based on findings from various studies indicating a correlation between the features and MS progression. Lesion count and load are used as measures for disease activity and predictors for the degree of disease progression [7]. Lesion location and distribution has been found to be an important factor as well [8]. Older patients are known to develop more severe disabilities than younger ones with the same T2 lesion load [9], and males are more likely to develop long-term disabilities compared to females [10]. However, it is not known how these features perform in classification tasks and, especially, which combinations of these features have the highest predictive capacity. Therefore, all possible combinations of the 15 features were iteratively tested towards their classification accuracy with a leave-oneout cross-validation (LOO-CV). The gold standard for the classification task was the occurence of a second clinical attack within one year. 

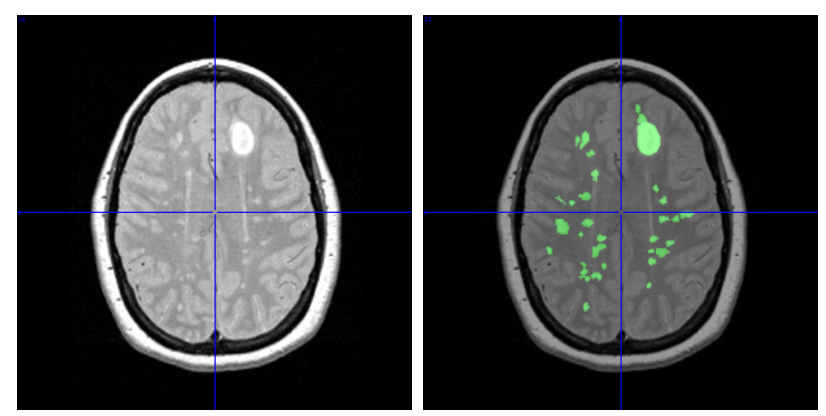

Figure 1. PD-weighted MRI scan with hyperintense lesions (a) and the same scan overlayed with a lesion mask (b).

Table I

SUMMARY OF RADIOLOGICAL, CLINICAL AND DEMOGRAPHIC PATIENT INFORMATION.

\begin{tabular}{l|l} 
Number of patients & 73 \\
\hline Gender $(\mathrm{m} / \mathrm{f})$ & $27 / 46$ \\
\hline Age in years (mean [range]) & $34.3[19.4-49.8]$ \\
\hline EDSS (median [range]) & $1[0-8]$ \\
\hline Converters at 1 year & $22 / 73(30 \%)$ \\
\hline Lesion load in $\mathrm{mm}^{3}($ mean [range]) & $2285.1[29-22581]$ \\
\hline Lesion count (median [range]) & $11[1-108]$
\end{tabular}

\section{A. Patient data}

73 patients were presented in London with isolated syndromes in the optic nerve, brainstem and spinal cord. They were scanned during the acute or subacute phase on a 1.5 T GE Signa MRI system. The protocols included T1weighting and dual spin echo for T2- and PD-weighting. A single neurologist masked the lesions on basis of the PDweighted images and using the $\mathrm{T} 2$ scans as a reference. A summary of the radiological, clinical and demographic patient information is presented in Table I.

\section{B. Feature Calculation}

15 features were used for the classification. Two of them are clinical: onset location and EDSS [11]; two are demographic: age and gender; and 11 are lesional features calculated from the MRI scans and binary lesion masks. The following list gives a detailled explanation of the individual features used for this study.

1) Lesion Count: Number of WM T2 lesions calculated from the binary lesion masks using an 18-connected neighbourhood - Indicates severity of lesion occurance

2) Lesion Load: Total volume of WM T2 lesions in $\mathrm{mm}^{3}$ calculated from the lesion masks - Indicates severity of lesion occurance

3) Mean Lesion Size: Lesion Load / Lesion Count Indicates severity of lesion occurance

4) Standard Deviation of Mean Lesion Size - Describes variation in individual lesion sizes

5) Minimal Lesion Size: Smallest lesion in patient in $\mathrm{mm}^{3}$ using an 18-connected neighbourhood

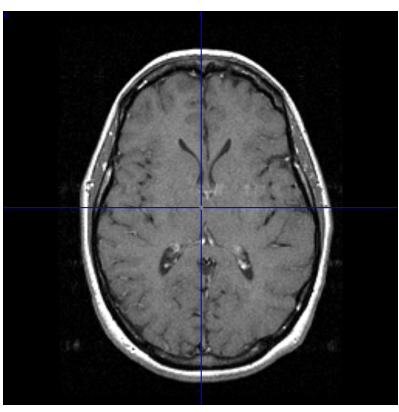

(a)

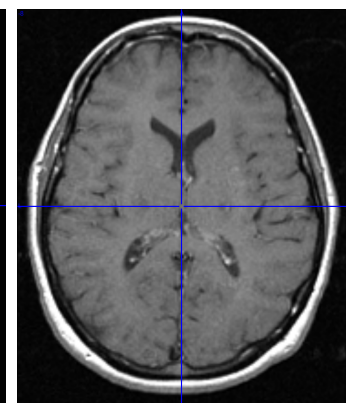

(b)
Figure 2. Misaligned images make it difficult to estimate the centre of the brain (a). Rigid registrations shift and resize the image to match a template with a known centre without the introduction of a strong bias (b).

6) Maximal Lesion Size: Largest lesion in patient in $\mathrm{mm}^{3}$ using an 18-connected neighbourhood

7) Age: Patient age in years (arithmetically rounded)

8) Gender: Male or Female - Indicates risk for MS

9) Onset Location: Location of first clinical attack (spinal cord, optic nerve, brainstem, multifocal)

10) EDSS: Expanded Disability Status Scale - Disability scale for MS in steps of 0.5 from 0 (normal neurological exam) to 10 (death due to MS)

11) Brain Volume: Total volume of brain tissue voxels (no cerebrospinal fluid) obtained with brain extraction tool (BET) [12]

12) WM Volume: Volume of white matter voxels (Segmentation threshold at 51\%)

13) GM Volume: Volume of gray matter voxels (Segmentation threshold at $51 \%$ )

14) Mean Distance to Brain Centre: Distance from lesion centroid to central voxel of MNI-152 template

15) Standard Deviation of Mean Distance to Brain Centre

\section{Image Processing}

For this study the images were modified as little as possible in order to avoid any bias occuring from the individual image processing steps like e.g. a change of lesion load after a non-linear registration. However, there were inavoidable steps. First, the patients' heads were not necessarily perfectly centered in the MRI scanner so that we performed a ridig registration to the MNI-152 template using NiftyReg [13] in order to measure the distances between the lesion centroids and the centre of the brain which was here defined as the central voxel of the MNI-152 template (Figure 2).

Second, it is a known problem that lesions affect the segmentation of white matter as they appear hyperintens in T2- and PD-weighted MRI, and iso- or hypointens in T1weighted MRI. Therefore, most algorithms classify lesions as being part of cerebrospinal fluid (CSF) so that the white matter segmentation has 'holes' and generally the WM volume is underestimated. A common method to overcome this is lesion filling as proposed by [14] for T1-weighted 


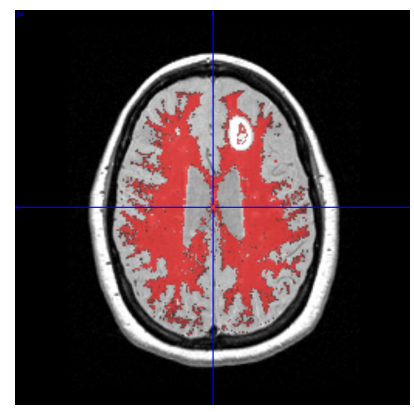

(a)

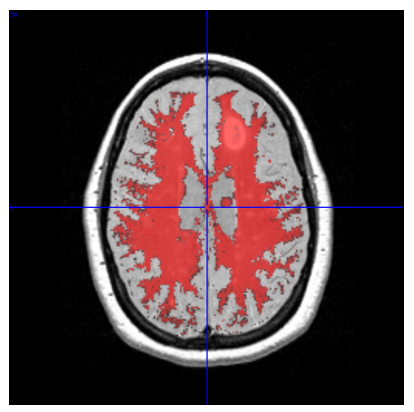

(b)
Figure 3. Hyperintense lesions lead to gaps in the WM segmentation and therefore an underestimated WM volume (a). The introduction of a lesion prior is a possible solution for this problem in low-contrast data (b).

MRI. However, this histogram-based method requires highcontrast images in order to differentiate between WM and GM - a requirement that was not sufficiently fulfilled in the present data set. Therefore, a tissue probality map for lesions was created using the binary lesion masks and smooth them with a gaussian kernel of size $1 \mathrm{~mm}$. Finally, all patients' brains were segmented using NiftySeg [15] with four tissue probability atlases for WM, GM, CSF and lesions. As it is known that the present lesions are all WM lesions the two resulting probabilistic segmentations for WM and lesions could be added resulting in an improved WM segmentation (Figure 3).

\section{Classification}

The classification of converters from CIS to CDMS and non-converters was performed using the library for support vector machines LIBSVM [16] and a leave-one-out cross-validation (LOO-CV). SVMs were originally designed to perform linear binary classification tasks such that the margin between the two classes is maximised [17]. The data points that are closest to the classification border are referred to as support vectors. However, real data usually is not sufficiently clustered and therefore cannot be linearly seperated. Non-linear classifcation becomes possible by applying kernel functions to the data and map it into a higherdimensional feature space which is less dense and possibly allows the calculation of a hyperplane that finally seperates the two classes.

Commonly used kernel functions are

- Linear Kernel: $k_{\text {Lin }}=\mathbf{u}^{\prime} * \mathbf{v}$

- Polynomial Kernel: $k_{\text {Poly }}=\left(\gamma \mathbf{u}^{\prime} * \mathbf{v}\right)^{d}$

- Radial Basis Function (RBF): $k_{\mathrm{RBF}}=e^{-\gamma|\mathbf{u}-\mathbf{v}|^{2}}$

with $\mathbf{u}$ and $\mathbf{v}$ being feature vectors, and $\gamma$ and $d$ parameters that have to be estimated.

SVMs belong to the group of supervised learners so only a part of the data is used to train the classifier, learn properties, and find the seperating hyperplane. Once this plane is found new, previously unseen data can be applied and classified.
Using LOO-CV all but one data point is used for training and the remaining point for testing. Training and testing points are then permuted until every patient has been used for testing once.

\section{EXPERIMENTS}

The aim of this study was to identify the feature combinations that have the highest capacity for the prediction of a second clinical attack and, hence, the diagnosis of clinically definite multiple sclerosis. In order to do this all possible combinations of the 15 features were used as an input for the SVM resulting in $2^{15}-1=32767$ feature combinations ( -1 for the combination with no features). Every combination has been tested for different parameter combinations. In particular, $\gamma$ and the cost parameter $c$ have been varied as multiples of two from $2^{-1}$ to $2^{8}$ resulting in a total of $32767 * 10 * 10=3,276,700$ experiments. This has been run for both the ploynomial and the RBF kernel with a LOO-CV. Since the present data set consists of data from 22 converters but 51 non-converters, it was not possible to use all patient data at once as this would lead to imbalanced groups and therefore bias the sensitivity and specificity measures. Therefore, the non-converter group was randomly split into three sub-groups of 22 patients to match the size of the converter group. Finally all experiments have been run for all three 22 vs 22 pairs in MATLAB [18].

\section{RESULTS}

The results of two different kernel functions are presented, those from the polynomial kernel $\left(\mathrm{SVM}_{\text {Poly }}\right)$ and those obtained from the RBF kernel $\left(\mathrm{SVM}_{\mathrm{RBF}}\right)$.

The $\mathrm{SVM}_{\text {Poly }}$ correctly classified up to 38 out of 44 patients, giving an accuracy of $86.36 \%$. The sensitivity was $81.82 \%$, the specificity $90.91 \%$, the positive predictive value (PPV) $90.00 \%$ and the negative predictive value (NPV) $83.33 \%$. The results of the $\mathrm{SVM}_{\mathrm{RBF}}$ were comparable with also 38 correctly classified patients, an accuracy of $86.36 \%$, and a sensitivity/specificity/PPV/NPV of $86.36 \% / 86.36 \% / 86.36 \% / 86.36 \%$.

The feature combinations leading to this results are shown in Table II.

It can be seen that the features Gender and Onset Location appear in all important feature combination indictaing a key role in the prediction of conversion from CIS to CDMS. However, these features alone only provide an accuracy between $52 \%$ and $66 \%$ so that it is necessary to support them by other features as e.g. shown in Table II.

The two distance-based measures Mean Distance to Brain Centre and its Standard Deviation of Mean Distance are not used in any of the highly predictive combinations and therefore seem to be not useful for this approach. 
Table II

OVERVIEW OF FEATURES COMBINATIONS LEADING TO THE HIGHEST CLASSIFICATION ACCURACIES FOR BOTH THE POLYNOMIAL KERNEL AND THE RADIAL BASIS FUNCTION.

\begin{tabular}{l|c|c|c|c|c|c} 
Features & \multicolumn{3}{|c|}{ SVM } & \multicolumn{3}{|c}{ SVM $_{\text {RBF }}$} \\
\hline Lesion Count & 1 st & 2nd & 3rd & 1 st & 2nd & 3rd \\
Lesion Load & $\bullet$ & & & $\bullet$ & & $\bullet$ \\
Mean Size & & $\bullet$ & & & & \\
Std of Mean Size & & $\bullet$ & $\bullet$ & & $\bullet$ & \\
Min Size & & $\bullet$ & & $\bullet$ & & $\bullet$ \\
Max Size & & $\bullet$ & & & & $\bullet$ \\
Age & $\bullet$ & $\bullet$ & $\bullet$ & $\bullet$ & & \\
Gender & $\bullet$ & $\bullet$ & $\bullet$ & $\bullet$ & $\bullet$ & $\bullet$ \\
Onset Location & $\bullet$ & $\bullet$ & $\bullet$ & $\bullet$ & $\bullet$ & $\bullet$ \\
EDSS & & & & & & $\bullet$ \\
Brain Volume & $\bullet$ & & $\bullet$ & & $\bullet$ & $\bullet$ \\
WM Volume & & & $\bullet$ & & & \\
GM Volume & & $\bullet$ & & $\bullet$ & $\bullet$ & \\
Mean Dist to Brain Centre & & & & & & \\
Std of Mean Distance & & & & & & \\
\hline Accuracy in \% & 86 & 86 & 84 & 86 & 86 & 84 \\
Sensitivity in \% & 82 & 82 & 77 & 86 & 86 & 86 \\
Specificity in \% & 91 & 91 & 91 & 86 & 86 & 82 \\
PPV in \% & 90 & 90 & 89 & 86 & 86 & 83 \\
NPV in \% & 83 & 83 & 80 & 86 & 86 & 86
\end{tabular}

\section{CONClusion}

It has been shown that the new features can be used in an SVM to predict a second clinical attack in CIS patients, and the accuracies for this prediction have been improved compared to previous studies. The obtained accuracies of up to $86.4 \%$ are competitive and in a range of clinical usability.

However, the feature definitions are not yet optimal and there is room for improvement. Further work will investigate more useful measures for lesion distribution in order to replace the two distance-based measures that did not support the classification in this study.

The segmentation, especially of the white matter, may be improved with new tools for lesion filling, and images with a higher contrast and signal-to-noise ratio. A possible solution for lesion filling is inpainting as presented by [19].

Finally, the findings have to be validated with another data set to prove the general applicability.

\section{REFERENCES}

[1] Marvin M. Goldenberg, Multiple Sclerosis Review, Pharmacy \& Therapeutics, Vol. 37(3), pp. 175-184, 2012

[2] David H. Miller, Jürg Kesselring, W. Ian McDonald et. al, Magnetic Resonance in Multiple Sclerosis, Cambridge University Press, 1997

[3] Charles M. Poser, Donald W. Paty, Lab Scheinber et. al, New Diagnostic Criteria for Multiple Sclerosis: Guidelines for Research Protocols, Annals of Neurology, Vol. 13(3), pp. 227231,1983

[4] David H. Miller, Declan T. Chard and Olga Ciccarelli, Clinically Isolated Syndromes, The Lancet Neurology, Vol. 11, pp. 157-169, 2012
[5] Philip P. Kwok, Olga Cicarelli, Declan T. Chard et. al, Predicting Clinically Definite Multiple Sclerosis from Onset using $S V M$, Machine Learning and Interpretation in Neuroimaging, Vol. 7263, pp. 116-123, 2012

[6] Olga Ciccarelli, Philip P. Kwok, Viktor Wottschel et. al, Predicting Conversion to MS in Patients with CIS using MachineLearning Techniques, ECTRIMS, No. 113, 2012

[7] S. P. Morrissey, David H. Miller, B. E. Kendall et. al, The Significance of Brain Magnetic Resonance Imaging Abnormalities at Presentation with Clinically Isolated Syndromes Suggestive of Multiple Sclerosis: A 5-year Follow-up Study, Brain, Vol. 116(1), pp. 135-146, 1993

[8] Benedetta Bodini, Marco Battaglini, Nicola De Stefano et. al, T2 lesion location really matters: a 10 year follow-up study in primary progressive multiple sclerosis, Journal of Neurology, Vol. 82, pp. 72-77, 2011

[9] Richard A. Rudick, Jar-Chi Lee, Jack Simon et. al, Significance of T2 Lesions in Multiple Sclerosis: A 13-year Longitudinal Study, Annals of Neurology, Vol. 60(2), pp. 236-242, 2006

[10] Zhaleh Khaleeli, Olga Ciccarelli, Francesco Manfredonia et. al, Predicting Progression in Primary Progressive Multiple Sclerosis: A 10-year Multicenter Study, Annals of Neurology, Vol. 63(6), pp. 790-793, 2008

[11] John F. Kurtzke, Rating Neurologic Impairment in Multiple Sclerosis: An Expanded Disability Status Scale (EDSS), Neurology, Vol. 33(11), pp. 14441452, 1983

[12] StephenM. Smith, Fast robust automated brain extraction, Human Brain Mapping, Vol. 17(3), pp. 143-155, 2002

[13] Marc Modat, Gerard R. Ridgway, Zeike A. Taylor, et. al, Fast Free-form Deformation using Graphics Processing Units, Computer Methods and Programs in Biomedicine, Vol. 98(3), pp. 278-284, 2009

[14] Declan T. Chard, Jonathan S. Jackson, David H. Miller et. al, Reducing the Impact of White Matter Lesions on Automated Measures of Brain Gray and White Matter Volumes, Journal of Magnetic Resonance Imaging, Volume 32(1), pp. 223-228, 2010

[15] Manuel J. Cardoso, Matt J. Clarkson, Gerard Ridgway et. al, LoAd: A Locally Adaptive Cortical Segmentation Algorithm, Neuroimage, Vol. 56(3), pp. 1386-1397, 2011

[16] Chih-Chung Chang and Chih-Jen Lin, LIBSVM: A Library for Support Vector Machines, ACM Transactions on Intelligent Systems and Technology, Vol. 2(3), pp. 27:1-27:27, 2011

[17] Vladimir N. Vapnik, The Nature of Statictical Learning Theory, Springer, New York, 1999

[18] MATLAB version 7.14.0.739 (2012a). Natick, Massachusetts: The MathWorks Inc., 2012

[19] Marco Battaglini, Mark Jenkinson, Nicola De Stefano, Evaluating and Reducing the Impact of White Matter Lesions on Brain Volume Measurements, Human Brain Mapping, Vol. 33(9), pp. 2062-2071, 2012 